\section{Cahiers de Narratologie}

Analyse et théorie narratives

$40 \mid 2021$

École, Récits, Nation

\title{
Le cinéma à l'aune de l'éducation nouvelle. Le point sur les pratiques du réseau Freinet en France dans les années 1920-1930.
}

\section{Leslie Dagneaux}

\author{
(2) OpenEdition \\ Journals \\ Édition électronique \\ URL : https://journals.openedition.org/narratologie/12565 \\ DOI : 10.4000/narratologie. 12565 \\ ISSN : 1765-307X \\ Éditeur \\ LIRCES
}

Référence électronique

Leslie Dagneaux, «Le cinéma à l'aune de l'éducation nouvelle. Le point sur les pratiques du réseau Freinet en France dans les années 1920-1930. », Cahiers de Narratologie [En ligne], 40 | 2021, mis en ligne le 06 décembre 2021, consulté le 25 décembre 2021. URL : http://journals.openedition.org/ narratologie/12565; DOI : https://doi.org/10.4000/narratologie.12565

Ce document a été généré automatiquement le 25 décembre 2021.

Article L.111-1 du Code de la propriété intellectuelle. 


\title{
Le cinéma à l'aune de l'éducation nouvelle. Le point sur les pratiques du réseau Freinet en France dans les années 1920-1930.
}

\author{
Leslie Dagneaux
}

\section{Introduction}

1 En France, les années 1920-1930 marquent les débuts du cinéma à l'école : rapport officiel, littérature critique, organismes étatiques et structures associatives témoignent de l'activité que génèrent la réflexion et l'organisation du cinéma pédagogique ${ }^{1}$. Le cinéma à l'école est alors essentiellement lié à la projection de films. Mais l'étude des pratiques en usage dans les milieux alternatifs d'éducation montre que, pour une petite partie de la population d'écoliers français, le cinéma à l'école est aussi lié, à la même période, à la réalisation de films. C'est le cas parmi les classes inscrites dans le réseau Freinet $^{2}$. Contrairement aux autres réseaux d'éducation nouvelle, le réseau Freinet est implanté dans le système public d'éducation. De la fin des années $1920^{3}$ à la fin des années 1930, il réunit plusieurs centaines d'éducateurs autour de la figure de Célestin Freinet, un jeune instituteur du sud-est de la France qui, au début des années 1920, développe de nouvelles techniques d'apprentissage en introduisant l'imprimerie dans sa classe.

2 Le réseau Freinet constitue, en effet, un terrain privilégié pour observer des pratiques novatrices du cinéma à l'école car c'est l'un des seuls réseaux alternatifs d'éducation en France à avoir autant pensé et organisé, à l'époque, la question du cinéma pédagogique. Ces initiatives passées, même si elles n'ont concerné que peu de classes et n'ont pas dépassé le stade expérimental, ont, en effet, donné lieu à des expérimentations hors du commun et des réflexions pédagogiques très approfondies en leur temps mais dont peu a été dit jusqu'ici. La présente synthèse issue de recherches menées dans le cadre d'une 
thèse d'histoire du cinéma ${ }^{4}$, élargit la connaissance de l'histoire des représentations du cinéma à l'école en montrant combien l'éducation nouvelle a pu représenter un vecteur d'innovation dans l'exploitation pédagogique du cinéma et constituer une force de proposition pour repenser les formes et les modalités d'existence du cinéma à l'école.

Si la réalisation de films en contexte scolaire est particulièrement rare dans les années 1920-1930 et suscite rétrospectivement des interrogations par rapport aux motivations pédagogiques, les expériences liées à l'emploi de la projection animée comme moyen d'enseignement intriguent par leur originalité. Mais le plus intéressant est de voir, audelà du particularisme des pratiques expérimentées, comment les praticiens du réseau Freinet se sont emparés du cinéma et ont développé une pédagogie cohérente. Ici, les pratiques liées à la projection et la réalisation misent sur une autre économie du film à l'école : sur le plan pédagogique, avec la réflexion conjointe sur les outils du cinéma et les dispositifs d'apprentissage ; sur le plan idéologique, avec la réflexion sur la place et le rôle du cinéma. L'article aborde en premier lieu la pratique de la projection animée comme moteur et source d'activité libre. Il s'agit de voir comment la pédagogie du «faire par soi-même» s'applique aux praticiens du réseau Freinet qui ont pensé l'introduction de cet outil moderne dans leur classe. Le second point traite de la réalisation de films dans ce cadre et pose la question de sa pertinence pédagogique. Ces pratiques pédagogiques renvoient à des manières de voir et/ou de faire des films, et donc, à une pratique sociale du cinéma, qui sera, pour finir, mise en perspective avec le projet politique dans lequel elle s'inscrit.

\section{La projection animée, moteur et source d'activité libre en classe}

4 L'éducation nouvelle introduit l'idée d'un apprentissage vivant qui induit une participation active de l'individu. Dans les années 1920-1930, sous l'influence de l'éducation nouvelle, la projection animée est intégrée comme un élément de pédagogie active notamment pour éveiller l'intérêt des élèves et susciter l'échange en classe ${ }^{5}$. Ainsi est née la leçon-filmée, procédé qui consiste à illustrer la leçon orale par le visionnement (simultané ou non) d'un film. Mais dans le milieu de l'éducation nouvelle, et au sein du réseau Freinet en particulier, le procédé ne convainc qu'à moitié. La leçonfilmée, si elle désenclave la parole de l'enseignant, s'inscrit, malgré tout, dans un cadre d'enseignement où l'enseignant tient une place dominante dans l'organisation et la transmission du savoir. Les classes du réseau Freinet adoptent un fonctionnement différent. Dès le milieu des années 1920, Célestin Freinet ne fait plus de leçons et privilégie le travail individuel ou en groupe : les découvertes dans le domaine de la science de l'enfant ont mis en évidence son «besoin de faire ». Au fur et à mesure des années, les membres du réseau Freinet développent donc une série d'outils et de techniques allant dans le sens de l'autonomie de l'élève. Des moyens sont mis à l'étude pour favoriser sa liberté d'action dans le cadre de l'utilisation pédagogique de la projection animée.

Ce qui caractérise l'action du réseau Freinet en matière d'usage de la projection animée dans le cadre scolaire, c'est que le champ de ses recherches, qui tend à dépasser l'usage courant du film comme simple illustration, a pour objectif premier l'organisation d'un cadre d'enseignement moins formel où il n'y a pas à proprement parler de «leçon ». On change de paradigme: le film n'est pas que l'outil de l'enseignant mais aussi celui de 
l'élève, et la projection n'est pas uniquement collective : elle peut être individuelle afin de répondre aux besoins singuliers de chaque enfant. Elle prend ainsi la forme d'une activité libre dont le fonctionnement repose sur le principe d' « auto-pmotivation » de l'élève. Cela commence dès le choix du film. La classe gère elle-même la commande des films qu'elle choisit (les membres du réseau Freinet ont leur propre cinémathèque). Le film est étudié par l'élève, seul ou en petit groupe, puis projeté collectivement durant les exposés. Un compte-rendu du film est parfois rédigé. Il ne s'agit pas d'un exercice d'application imposé : ce travail est motivé par le désir de l'élève de s'exprimer. On manque malheureusement de renseignements sur les conditions de projection (salle, aménagement, horaire) et sur le rôle de l'enseignant dont on suppose qu'il s'occupait au moins de l'aspect technique. En effet, si les films utilisés sont de petit format $(9,5 \mathrm{~mm})$ et les bobines beaucoup plus légères que pour le format standard $(35 \mathrm{~mm})$, leur installation requiert une certaine dextérité. On ne sait pas non plus dans quelle mesure l'enseignant apportait son aide à l'élève pour mieux comprendre le propos du film. Cet aspect pose en tout cas problème et fait, à l'époque, l'objet d'une réflexion parallèle sur les outils à mettre au point pour accompagner la projection.

Dans les années 1920-1930, le marché du film scolaire est en pleine ébullition. Les films projetés en classe sont des remontages de documentaires préexistants mais il existe aussi de plus en plus de films tournés exprès, notamment par des enseignants ${ }^{6}$. Les films d'enseignement sont conçus comme des aides à la leçon. Particulièrement démonstratifs, ils suivent un cheminement explicatif déterminé en vue d'un apprentissage ciblé. Ceux qui remplacent l'observation et l'expérimentation directe sont critiqués par les praticiens de l'éducation nouvelle. Mais pour certains enseignements comme la géographie ou les sciences naturelles, ils sont plébiscités parce qu'ils ouvrent une fenêtre sur le monde. Ces films sont aussi utilisés au sein du réseau Freinet. Outre les expériences sur le visionnement autonome du film, des recherches sur la conception d'outils documentaires vont déboucher sur la création de fiches papier documentées abordant des sujets divers, que les élèves consultent à l'occasion de leurs travaux. Parmi elles, les «documents-cinéma» présentent un résumé détaillé du contenu du film. Dans quelques cas, ils apportent un prolongement avec des renseignements complémentaires et des références bibliographiques.

7 L'interaction entre l'élève et le film est centrale dans la réflexion sur l'utilisation pédagogique du cinéma au sein du réseau Freinet. Elle passe par une prise de contact directe avec l'outil. La prise en compte du rôle que l'enfant peut jouer quand il n'est pas réduit à un simple consommateur d'images conduit à des expérimentations plus poussées pour trouver le moyen d'impliquer davantage l'élève dans l'usage du cinéma. Dans une pédagogie du «faire » qui se définit par l'action de l'élève, la réalisation de films s'impose naturellement. Mais pourquoi réaliser des films en classe et en quoi servent-t-ils l'apprentissage scolaire?

\section{La correspondance filmée, nouveau moyen d'enseignement par le film}

Dans les années 1920-1930, la caméra n'est pas admise par l'institution scolaire comme outil pédagogique. Les campagnes de sensibilisation au cinéma scolaire visent à promouvoir l'équipement pour la projection en classe. Si elles sont le fruit d'initiatives individuelles qui ne sont pas vraiment reconnues en dehors d'un cercle d'initiés, les 
expérimentations timides dans le milieu de l'éducation nouvelle témoignent néanmoins d'un intérêt manifeste pour la pratique du cinéma à des fins éducatives. Les praticiens du réseau Freinet - Célestin Freinet en tête - figurent parmi les pionniers, en France, de l'introduction de la caméra dans la classe. À l'époque, il ne s'agit pas d'initier les élèves à la réalisation de films dans une perspective d'éducation cinématographique. Dans les classes du réseau Freinet des années 1920-1930, la pratique du cinéma répond à un besoin de films. Les films d'enseignement du marché présentent un savoir constitué (et institué) qui ne satisfait pas la curiosité que l'enfant manifeste pour tout ce qui l'entoure et qui n'est pas au programme scolaire.

9 L'histoire de la réalisation de films dans les classes du réseau Freinet est liée, au départ, à la pratique de la correspondance filmée qui va donc fournir le cadre de ces réalisations. L'idée est née avec l'imprimerie, les films venant compléter la correspondance écrite. Cette pratique crée les conditions d'une interaction naturelle entre l'élève et le film dans la mesure où le film fait partie intégrante de la vie de la classe. En effet, l'éducation nouvelle ne s'intéresse pas seulement aux modalités de transmission, elle travaille aussi la question des savoirs et de leur réévaluation au regard notamment des centres d'intérêt des élèves.

10 La plupart des films échangés dans les années 1920-1930 - une vingtaine - sont perdus. Voici quelques titres: Fabrication de parfum aux environs de Cannes, La culture de l'olive et l'industrie de l'olive, Les élèves de Bar-sur-Loup au travail, La classe de Trégunc Saint-Philibert à la pointe de Trévignon. Ce dernier est devenu emblématique de cette pratique pédagogique alors émergente : il est en accès libre sur internet ${ }^{7}$. D'une durée d'environ deux minutes, il présente l'arrivée des parents pêcheurs au port accueillis par les élèves, fait défiler les correspondants bretons un à un devant la caméra, et finit sur un effet « carte postale » de paysages maritimes. Ces petites productions muettes tournées en noir et blanc au format 9,5 $\mathrm{mm}$ adoptent plus la forme du reportage que du film d'enseignement. La vie constitue en le thème principal : certains présentent les élèves dans des situations variées (travail, jeux, sorties scolaires) tandis que d'autres illustrent l'environnement géographique de la classe (particularités régionales). Si ce sont les adultes qui filment ${ }^{8}$, les enfants participent au choix du sujet et à l'élaboration du synopsis. Plus ou moins instructifs, tous ces films tirent leur valeur pédagogique de la correspondance qui les a suscités. Ce qui importe dans cette démarche, c'est surtout l'acte d'échanger. La correspondance filmée constitue un moyen de vivifier l'apprentissage car elle enrichit les expériences humaines, de communication notamment. Mais la priorité donnée à l'outil semble engager des exigences moindres au niveau de la transmission des savoirs scolaires. Ce mode d'apprentissage privilégie non plus la connaissance à acquérir mais ce qui est à la base de la vie de l'enfant, c'est-à-dire le travail qu'il réalise ou dont il est spectateur.

11 La projection et la réalisation de films dans le cadre de la correspondance filmée instaurent un rapport de proximité inhabituel entre l'élève et le film qui pose question au regard de sa posture et de ses habitudes de spectateur de cinéma. Car l'école est aussi un lieu d'accès au cinéma. L'intégration du cinéma à l'école participe d'une logique de vulgarisation qui n'est pas uniquement liée à la défense d'un accès égal à la connaissance et aux moyens d'y parvenir, mais qui relève également de la démocratisation du cinéma comme activité de loisir. La correspondance filmée introduit une autre culture du cinéma que le discours politique des praticiens du réseau Freinet permet d'éclairer. 


\section{Le cinéma, outil d'émancipation culturelle et politique} Freinet, en l'occurrence, inscrit son projet pédagogique dans un projet, plus vaste, de société. Ses pratiques sont très ancrées dans l'idéologie communiste. Et pour cause : les membres du réseau Freinet, durant ses premières années d'existence, sont pour la plupart syndiqués à la Fédération de l'Enseignement Unitaire ${ }^{9}$. L'introduction du cinéma en classe comme outil de communication ne peut pas, de fait, être dissociée du discours politique de ces praticiens ni du contexte socio-économique de l'époque ${ }^{10}$. D'une part, les praticiens du réseau Freinet rejettent le modèle capitaliste et sont relativement critiques vis-à-vis des évolutions techniques et culturelles qui $\mathrm{y}$ participent. Les films commerciaux sont associés à un cinéma de profit, jugé antiéducatif par essence. D'autre part, les années 1920-1930 voient la montée des extrémismes et le développement du cinéma de propagande. L'image est perçue comme un instrument de pouvoir et de manipulation. Les praticiens du réseau Freinet impliqués dans la vie politique prennent conscience, à ce moment, que le cinéma peut asservir l'individu et s'en méfient, mais, d'un autre côté, ils veulent l'utiliser pour servir la cause de la libération de l'individu par lui-même ${ }^{11}$.

Chez les praticiens du réseau Freinet, la démarche consistant à introduire le cinéma dans l'enseignement par le moyen de la correspondance filmée s'accompagne d'une volonté de s'emparer des moyens de production de la culture. Il ne s'agit pas de " démocratisation culturelle». Chez eux, faire du cinéma une pratique populaire ne consiste pas à ne montrer que des grands films. Leur conception du cinéma ne renvoie pas à une définition savante de la culture mais englobe la problématique de l'amateur. Freinet et ses camarades du mouvement veulent un cinéma proche du peuple, c'est-àdire des films dont il est à la fois le sujet et l'acteur, ce qui implique sa présence à l'écran. Les films réalisés avec peu de moyens par des non-spécialistes initient un rapport intime au cinéma parce qu'ils touchent à la réalité de l'environnement des spectateurs. Mais la correspondance filmée participe surtout d'une logique d'appropriation du cinéma dans la mesure où l'individu - l'élève - est en contact avec l'outil de création et participe à la fabrication du film.

14 L'histoire de l'introduction de la caméra dans les classes qui la pratiquent a donc aussi à voir avec l'idée d'instaurer un rapport intime au cinéma en familiarisant l'élève avec le cinéma « en train de se faire ». Il s'agit, en l'occurrence, de l'émanciper de son rôle primaire de spectateur par la pratique et de le faire accéder à une dimension supérieure du cinéma en tant qu'instrument de langage accessible à tous. L'école n'est pas uniquement un lieu où l'on voit des films. C'est aussi l'endroit idéal pour transformer un media conçu initialement comme objet de consommation en outil d'éducation à la créativité. Les caméras qui s'invitent dans les classes Freinet opèrent une révolution : les élèves découvrent le cinéma autrement

Chez les praticiens du réseau Freinet, la pédagogie du «faire » s'oppose à toute forme de domination. L'échange de films impulse, de fait, une autre économie du cinéma avec son propre mode de production (filmeurs non professionnels, sujets familiers, petit budget, décor du quotidien) et son propre réseau de diffusion (les écoles). Cette pratique met ainsi en jeu d'autres modèles qui entrent en opposition avec l'utilisation commerciale et consommatrice du cinéma. 


\section{Conclusion} d'éducation nouvelle ne donne qu'un aperçu du contexte d'émergence de ces expérimentations et de leurs conditions de réalisation. Il ne saurait donc clore le sujet des pratiques alternatives et invite, au contraire, à mener d'autres recherches portant sur les autres réseaux d'enseignement alternatif, notamment à l'étranger, comme l'Angleterre où des expérimentations voisines ont lieu à la même époque. De même, si nous avons mis en lumière les innovations techniques et théoriques que le réseau Freinet contribua à rendre possibles, il nous faut rappeler que des pratiques similaires peuvent être observées ailleurs. Les expériences qui visent à faire de la projection un moteur et une source d'activité libre ne sont pas propres au «cercle Freinet »: on les retrouve chez les praticiens de la méthode Cousinet (travail libre par groupe) ou ceux de la méthode Decroly (exploitation des centres d'intérêt) ralliés au Groupe Français d'Éducation Nouvelle.

À plusieurs égards, l'éducation nouvelle et le réseau Freinet offrent un terrain d'observation pour des recherches plus poussées, notamment en ce qui concerne les pratiques cinématographiques des jeunes que le cadre des années 1920-1930 n'a pas permis d'aborder ici. La pratique du cinéma par les jeunes se développe effectivement plus tard, après la Seconde Guerre mondiale, avec l'émergence de l'éducation cinématographique où, là encore, l'éducation nouvelle a été influente. On pense aussi aux productions nées de ces pratiques qui intéressent un champ des études cinématographiques encore inexploré. Le sujet de l'éducation nouvelle et de ses relations avec le cinéma ouvre ainsi des perspectives de recherche plus vastes qui rejoignent les préoccupations actuelles des chercheurs en cinéma, lesquelles accordent une plus grande attention aux pratiques marginales et non conventionnelles.

\section{BIBLIOGRAPHIE}

Ariès, Paul. « Le cinéma éducateur dans les années trente ou la laïcité au service du cinéma », $1895, n^{\circ} 14$, juin 1993, pp. 62-75.

Borde, Raymond ; Perrin, Charles. Les offices du cinéma éducateur et la survivance du muet (1925-1940). Lyon, Presses Universitaire de Lyon, 1992.

Bourdier, Philippe (dir.). Images à l'école, images de l'école. Actes du colloque organisé par le Musée de l'école d'Eure-et-Loir le 17 mars 2010. Chartres, Musée de l'école d'Eure-et-Loir, 2011.

Dagneaux, Leslie. Les techniques Freinet ou le cinéma scolaire autrement : l'émancipation comme maîtremot. Retour sur quarante ans d'expérimentations (1927-1968). Thèse d'histoire du cinéma, Université Rennes 2, 2017.

Gallinari, Pauline, Les communistes et le cinéma. France, de la libération aux années 60. Rennes, PUR, coll. « Histoire », 2015.

Cahiers de Narratologie, 40 | 2021 
Gauthier, Christophe. «Au risque du spectacle. Les projections cinématographiques en milieu scolaire dans les années $1920 »$ in De Pastre, Béatrice (dir.). Cinéma pédagogique et scientifique : à la redécouverte des archives. Lyon, ENS Éditions, coll. « Feuillets de l'ENS de Fontenay-Saint-Cloud », 2004, pp. 73-98.

Jeunet, Paul ; Nourrisson, Didier (dir.). Cinéma-École : aller-retour (Actes du colloque de SaintÉtienne, novembre 2000). Saint-Étienne, Publications de l'université de Saint-Étienne, 2001.

Portier, Henri. « À l'origine d'un mouvement pédagogique : des idées, des hommes...et le cinématographe » in Clanché, Pierre ; Debardieux, Éric ; Testanière, Jacques (dir.). La pédagogie Freinet : mises à jour et perspectives. Bordeaux, PUB, 1999, pp. 215-222.

Portier, Henri. « De l'utilisation du film comme outil pédagogique à l'appropriation du cinéma par les élèves comme outil de création ", in Jeunet, Paul ; Nourrisson, Didier (dir.). Cinéma-École : aller-retour (Actes du colloque de Saint-Étienne, novembre 2000). Saint-Étienne, Publications de l'université de Saint-Étienne, 2001, pp. 113-125.

Sentilhes, Armelle. « Le cinéma et les archives. L'audiovisuel au service de l'enseignement : projections lumineuses et cinéma (1880-1940) », La gazette des archives, n¹73, 1996, pp. 165-181.

Taillibert, Christel. L'Institut International du cinématographe éducatif: regard sur le rôle du cinéma éducatif dans la politique internationale du fascisme italien. Paris, L'Harmattan, coll. « Champs visuels », 1999.

Taillibert, Christel. «L'usage mixte de l'image fixe et de l'image animée dans le domaine de l'enseignement durant l'entre-deux-guerres » in Guido, Laurent ; Lugon, Oliver (dir.). Fixe/animé: croisements de la photographie et du cinéma au XX ème siècle. Lausanne, L'âge d'homme, coll. « Histoire et théorie du cinéma ", 2010, pp. 145-155.

Tixier, Jean-Marie. « Cinéma et école : petit flash-back historique », in De Luget, Agnès ; Flores, Magalie (dir.). L'enfant, le droit et le cinéma. Rennes, Presses Universitaires de Rennes, coll. «L'Univers des Normes », 2012, pp. 37-54.

Ueberschlag, Josette. Jean Brérault, l'instituteur cinéaste (1898-1973). Saint-Étienne, Publications de l'université de Saint-Étienne, 2007.

Vignaux, Valérie. Jean-Benoît Lévy ou le corps comme utopie : une histoire du cinéma éducateur dans l'entre-deux-guerres. Paris, AFRHC, 2007.

Vignaux, Valérie. «Célestin Freinet et le cinéma ou le cinéma français et l'éducation nouvelle (1927-1939) » in Bourdier, Philippe (dir.). Images à l'école, images de l'école (Actes du colloque organisé par le Musée de l'école d'Eure-et-Loir le 17 mars 2010). Chartres, Musée de l'école d'Eure-et-Loir, 2011, pp. 75-86.

\section{NOTES}

1. Voir notamment les études de Paul Ariès, Raymond Borde et Charles Perrin, et Jean-Marie Tixier. Pour une meilleure compréhension du contexte à l'échelle internationale, se reporter aux travaux de Christel Taillibert.

2. Les travaux d'Henri Portier sont les premiers à avoir mis en lumière ces pratiques : Portier, Henri. «À l'origine d'un mouvement pédagogique : des idées, des hommes...et le cinématographe » in Clanché, Pierre ; Debardieux, Éric ; Testanière, Jacques (dir.). La pédagogie Freinet : mises à jour et perspectives. Bordeaux, PUB, 1999, pp. 215-222 ; Portier, Henri. « De l'utilisation du film comme outil pédagogique à l'appropriation du cinéma 
par les élèves comme outil de création", in Jeunet, Paul ; Nourrisson, Didier (dir.). Cinéma-École : aller-retour (Actes du colloque de Saint-Étienne, novembre 2000). SaintÉtienne, Publications de l'université de Saint-Étienne, 2001, pp. 113-125.

3. Le réseau s'institutionnalise avec la création de la Coopérative de l'Enseignement Laïque en 1927.

4. Dagneaux, Leslie. Les techniques Freinet ou le cinéma scolaire autrement: l'émancipation comme maitre-mot. Retour sur quarante ans d'expérimentations (1927-1968). Thèse d'histoire du cinéma, Université Rennes 2, 2017.

5. Ces pratiques ont notamment été étudiées par Armelle Sentilhes et Christophe Gauthier: Sentilhes, Armelle. « Le cinéma et les archives. L'audiovisuel au service de l'enseignement : projections lumineuses et cinéma (1880-1940) ", La gazette des archives, n¹73, 1996, pp.165-181; Gauthier, Christophe. "Au risque du spectacle. Les projections cinématographiques en milieu scolaire dans les années $1920 »$ in De Pastre, Béatrice (dir.). Cinéma pédagogique et scientifique : à la redécouverte des archives. Lyon, ENS Éditions, coll. « Feuillets de l'ENS de Fontenay-Saint-Cloud », 2004, pp. 73-98.

6. Josette Ueberschlag et Valérie Vignaux ont consacré des ouvrages à deux auteurs de films d'enseignement emblématiques de l'époque: Ueberschlag, Josette. Jean Brérault, l'instituteur cinéaste (1898-1973). Saint-Étienne, Publications de l'université de SaintÉtienne, 2007 ; Vignaux, Valérie. Jean-Benoît Lévy ou le corps comme utopie : une histoire du cinéma éducateur dans l'entre-deux-guerres. Paris, AFRHC, 2007.

7. Le film est notamment consultable sur le site internet de l'association des amis de Freinet.

8. La caméra Pathé-Baby plébiscitée par les praticiens du réseau Freinet est légère et petite mais complexe à manipuler pour un enfant.

9. La Fédération de l'Enseignement Unitaire est rattachée à la Confédération Générale du Travail Unitaire reliée au Parti communiste français. L'organisation est minoritaire par rapport au puissant Syndicat National des Instituteurs.

10. Le travail mené par Pauline Gallinari sur les liens entretenus par les communistes avec le cinéma est, à cet égard, riche d'enseignements: Gallinari, Pauline, Les communistes et le cinéma. France, de la libération aux années 60. Rennes, PUR, coll. « Histoire », 2015.

11. Le réseau Freinet alimente ainsi la production de films en France comme le raconte Valérie Vignaux : Vignaux, Valérie. «Célestin Freinet et le cinéma ou le cinéma français et l'éducation nouvelle (1927-1939)» in Bourdier, Philippe (dir.). Images à l'école, images de l'école (Actes du colloque organisé par le Musée de l'école d'Eure-et-Loir le 17 mars 2010). Chartres, Musée de l'école d'Eure-et-Loir, 2011, pp. 75-86.

\section{RÉSUMÉS}

En France, les années 1990 révèlent un véritable intérêt des chercheurs pour l'histoire de l'image animée à l'école mais les pratiques pionnières liées à la projection et la réalisation de films sont encore peu analysées. Cet état des lieux prend appui sur un récent travail de thèse qui considère la question depuis l'un des plus grands réseaux français d'éducation nouvelle : le réseau Freinet. 
Tandis que les études sur les usages sociaux et la valeur culturelle du cinéma se multiplient, les découvertes de cette recherche mettent en lumière le terrain privilégié que représente l'éducation nouvelle pour observer des pratiques du cinéma à l'école hors du commun.

\section{INDEX}

Mots-clés : cinéma, école, éducation nouvelle, pratiques pédagogiques, projection, réalisation

\section{AUTEUR}

\section{LESLIE DAGNEAUX}

Docteure en Études CinématographiquesLe domaine de recherche de Leslie Dagneaux concerne les usages pédagogiques et sociaux du cinéma et, plus particulièrement, l'analyse des pratiques éducatives liées au cinéma. Ses travaux abordent les méthodes alternatives d'enseignement comme un vecteur d'innovation pour l'exploitation pédagogique du cinéma. Elle a soutenu en 2017 une thèse en histoire du cinéma intitulée « Les techniques Freinet ou le cinéma scolaire autrement : l'émancipation comme maître-mot. Retour sur quarante ans d'expérimentations (1927-1968) ». Elle est également l'auteure de deux études, l'une portant sur l'usage du film fixe chez les praticiens de la pédagogie Freinet, l'autre consacrée à l'action conjointe du mouvement Freinet et de la Ligue française de l'enseignement en matière de cinéma d'éducation populaire. Elle a, par ailleurs été amenée à travailler sur des questions liées à la couleur au cinéma ainsi qu’à la filmographie de Jean Epstein. 